\title{
Integración de mapas, fotos, videos, datos e historias en ArcGIS StoryMaps en proyectos de cursos de gestión de negocios internacionales.
}

Jose Gregorio Lepervanche Valencia

Florida State College at Jacksonville, jleperva@fscj.edu, jglepervanche@alum.mit.edu

\begin{abstract}
\$EWWFW
, QHLQDURQDO\%XVQHWO DQDJ H HQWFRXUHIQ WKH\%OFKHRUVR 6XSHULMRQDQGO DQDJ H HQWDQG

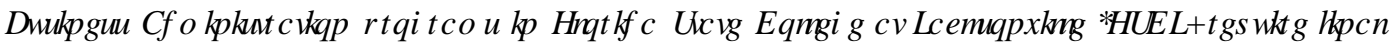
UHP SLRNFWW 6WGHQW KDYH UR FLHWHD IIFUMRXV FRQXXWQJ FRP SDQ DQG SLRGXFHD EXVQHW IQUTODJ HQFH USRLWRUD SUMHQUURQ IRUD IIFUMRXV FXWRP HU 7KH RENAFWYH RI WKH USRLWV IR

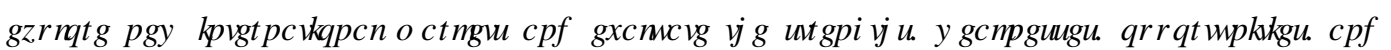

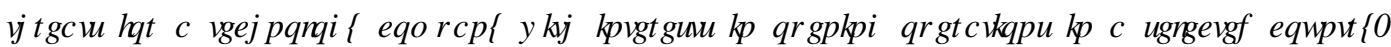

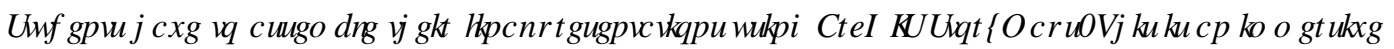

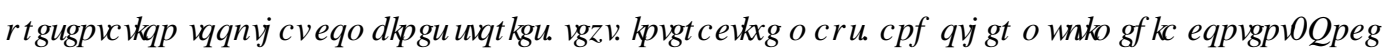

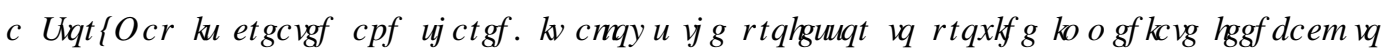

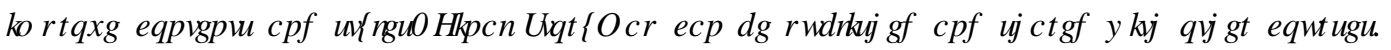
RUDQ]DURQV RUHHU RQH DURXQG UKH Z RLCH 7KIV USRLWXP P DUHH WKHULUWDOXVH IQ) 6\&-

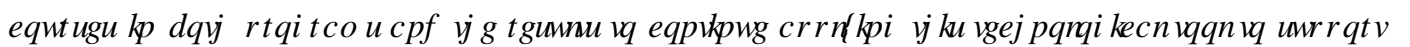

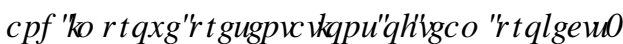

. HZRLGV , QHDFWHP DSV 6 LRU P DSV \%XYQHW, QWOJHFH5 HRUW, QHQDURQDO\%XVQHW 0 DQDJH HQW

\section{HXP HQ}

/ RV FXURV GH * HMHQ GH 1 HJRFIRV , QHLQDFIRQDON HQ ORV SURJUP DV GH / IFHQFDWWD HQ

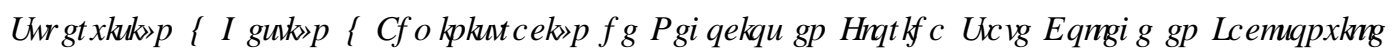
प) 6\&-口 UHXXIHHQ SUR HFURV GH HIXISR IIQDO / RV HMXGDQUN GEHQ FUHU XQD HPSUHD GH

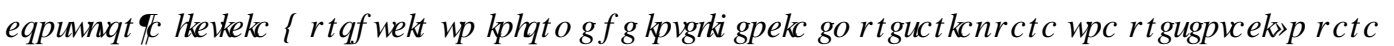

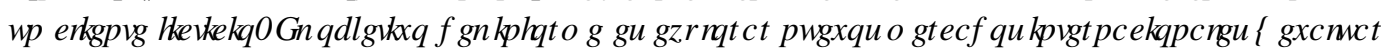

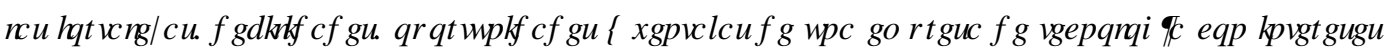
HQ DEUURSHDFIRQH HQXQSDVVHDFFIRQDOR] / RVHMXGDQWWGEHQRUDQIDUWVSUHMQWFIRQH

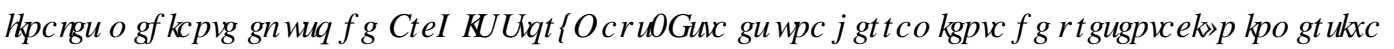

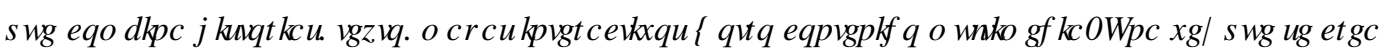

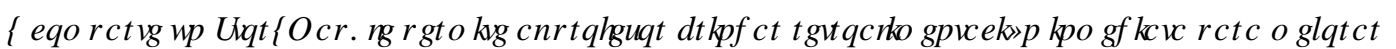
IRV FRQWDIGRV I HMCRV ( O6LRUO DS IIQDOVH SXHGH SXECFDU I FRP SDUWU FRQ RURV FXURV RUDQJDFIRQH R FXDTXIHSHURQD HQ WRR HOP XQRR ( WHIQRLP HUHXP HWV XVRVIQFHDONHQ (RVFXURV) 6\&- I XVUHXQDORVSDD VH XIUXUGD] DQCR HMDKHUDP IHQWDUFQRQ JIFDSDDDSR DU

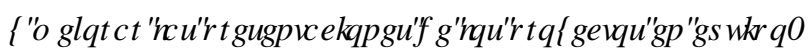

3 DOEWV FOVH PDSDV IQUADFURVI PDSDV FRQ KLMRUDV IQUHDJHQID GH QH RFIRV QHDRFIRV IQUAQDFIRQDAMUJHMH QGHQH RFIRV 
para proyectos de cursos de gestión de negocios internacionales.

\section{Introducción}

Este trabajo incluye un innovador recurso tecnológico para mejorar y dar a conocer las presentaciones de los proyectos finales de trabajo en equipo de los cursos de Gestión de Negocios Internacionales en los programas de Licenciatura en Supervisión y Gestión y Administración de Negocios en Florida State College en Jacksonville (FSCJ). Estos cursos requieren la elaboración de un reporte de inteligencia de negocios que integre los objetivos de aprendizaje específicos del curso. Esta es una herramienta de presentación inmersiva que combina historias, texto, mapas interactivos y otro contenido multimedia. Una vez que se crea y comparte un StoryMap, el profesor puede visualizar su contenido y progreso y brindar retroalimentación inmediata para mejorar los contenidos y estilos. Final StoryMap se puede publicar y compartir con otros cursos, organizaciones o cualquier persona en todo el mundo.

El proyecto final en el mencionado curso requiere que los estudiantes por equipos creen una empresa de consultoría ficticia a fin de producir un informe de negocios para una presentación para un cliente ficticio. El objetivo del informe es explorar nuevos mercados internacionales y evaluar las fortalezas, debilidades, oportunidades y ventajas de una empresa de tecnología con intereses en abrir operaciones en un país seleccionado. En el curso se analizan objetivos específicos (Gestión de mercadeo, posibles alianzas, distribución y mercado en Gestión de Negocios Internacionales). Los estudiantes deben organizar sus presentaciones finales utilizando únicamente ArcGIS StoryMaps y no presentaciones de PowerPoint o documentos en Word. Todo el contenido del informe, incluyendo referencias y nombres de los integrantes del equipo, debe ser incluido en el StoryMap.

\section{Objetivos}

Los objetivos del presente trabajo son los siguientes:

Introducir el uso de ArcGIS StoryMaps para la realización y presentación de proyectos de trabajo en equipo en cursos de Gestión de Negocios Internacionales.

Determinar los beneficios en el uso de ArcGIS StoryMaps para seguir el progreso de la elaboración del reporte y poder proveer retroalimentación inmediata a los estudiantes.

Utilizar el modelo de aceptación de tecnologías (Technology Acceptance Model) para determinar cómo los estudiantes llegan a aceptar y utilizar este recurso tecnológico para soportar su aprendizaje en los cursos seleccionados.

Compartir una selección de los StoryMaps publicados y los resultados del survey realizado al finalizar el curso de Gestión de Negocios Internacionales.

Expandir el uso de ArcGIS StoryMaps a otros cursos de estos programas que resaltan la necesidad de ubicar los países, ciudades y centros de producción distribución y logística como por ejemplo, Gestión Estratégica y Sistemas de Información Globales.

\section{Desarrollo de la innovación}

\subsection{Objetivos de aprendizaje del curso de Gestión de Negocios Internacionales.}


Florida State College at Jacksonville ofrece programas de Licenciatura en Supervisión y Gestión y Administración de Empresas. Ambos programas incluyen objetivos de aprendizaje relacionados con la gestión de negocios internacionales.. Ambos programas requieren que los estudiantes realicen simulaciones, escenarios, trabajos en equipo que incluya la investigación de gobiernos, empresas, y organizaciones internacionales.

El curso de Gestión de Negocios Internacionales estudia el proceso, la práctica y la teoría de los negocios internacionales. Los temas cubiertos incluyen, entre otros, sistemas financieros internacionales, comercio e inversiones internacionales y entornos comerciales interculturales. Los objetivos de aprendizaje son: 1. evaluar y discutir las prácticas de gestión desde una perspectiva internacional, 2. describir la gestión internacional, 3. el análisis estratégico y la planificación en un entorno global, 4. comprender la implementación y el control de un proyecto comercial internacional, y 5. analizar y discutir la gestión de los componentes tecnológicos para hacer frente a los desafíos de la globalización.

\subsection{ArcGIS Story Maps.}

Durante la búsqueda de innovaciones para para mejorar la enseñanza en los cursos de gestión internacional, se han incorporado diferentes modelos y herramientas tales como blogs, social media, presentaciones en Prezi, videos, y los recursos educativos de TED, TEDx y TED Ed (Lepervanche, 2018). Varios de los videos de TED y TEDx sirvieron como base para aprender acerca del uso de mapas, datos visuales geoespaciales y herramientas como OpenStreetMaps, Google Maps y ArcGIS StoryMaps. Al observar las nuevas herramientas para visualizar datos y mostrarlas en cursos, se encontró que las propuestas mostradas eran para profesionales en el tema con un alto grado de dificultad. Hwan (2017) mostró en su charla de TEDx algunos de los beneficios de utilizar mapas y tecnologías de visualización geoespacial de ciudades y datos. Al continuar la búsqueda de herramientas de mapas y data que fueran fáciles de utilizar por estudiantes de los programas de gestión de negocios, se observó la charla de McDermott (2017) donde visualizaba un gráfico de datos de la ciudad de Newton, North Carolina mediante el uso de ArcGIS Story Maps.

Al estudiar a ESRI y su herramienta ArcGIS Story Maps se observó que esta herramienta es ampliamente utilizada para desarrollar presentaciones que no requiere un conocimiento profundo de cartografía y que permite utilizar sus elementos para integrar imágenes, videos, gráficos con data, textos, enlaces web, y mapas. Según ESRI (2012) "los story maps combinan mapas con otros elementos que facilitan y enfatizan el mensaje que el creador busca transmitir. Título, texto, leyenda, ventanas emergentes y otros elementos visuales: gráficos, cuadros, fotografías, video, audio: ayuda a interpretar el mapa o los mapas que forman la pieza central de la historia". Al determinar que los Story Maps estaban destinados a un público no técnico y que servían para proveer información geográfica para informar, educar, entretener e involucrar a sus audiencias, el autor decidió elaborar Story Maps para su proyecto de Geolocalización de las Juderías de la Península Ibérica el cual, al combinar ArcGIS Story Maps con Google Maps ha servido para dar a conocer a estas juderías tal y como fue reportado en el periódico El Levante-El Mercantil Valenciano (Arribas, 2019).

El siguiente paso fue incorporar a ArcGIS StoryMaps como herramienta para el aprendizaje en los cursos de Gestión de Negocios y Sistemas de Información y se empezó una colección de Story Maps relacionados con algunos de los tópicos de estos cursos (Fig. 1) (https://arcg.is/15aeSu0). Al observar la aceptación y el interés en el uso de esta herramienta se tomó la decisión de incorporarlas a un primer proyecto en un curso piloto y se escogió al modelo de Aceptación Tecnológica TAC (Technology Acceptance Model) para determinar la aceptación de esta tecnología en los cursos de Gestión de 
para proyectos de cursos de gestión de negocios internacionales.

Negocios Internacionales ya que estos cursos utilizan una gran variedad de mapas, gráficos de datos y fotos de ciudades, oficinas, instalaciones, y rutas logísticas internacionales.

\section{COLLECTION \\ Dr Lepervanche Global Story Maps Campus}

Global Learning Maps used in courses of Management, Information Systems, and Cultural Tourism.

Get started

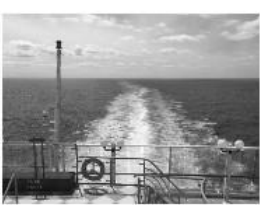

(1) Dr. Jose G. Lepervanche Valencia, Global Learning...

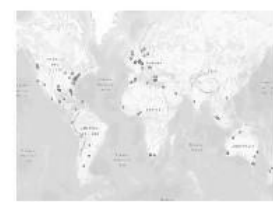

4. Global Sea Scouts \& Waterways, Global Maps \&...

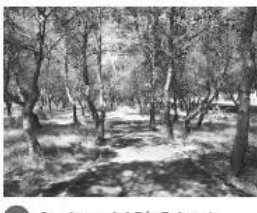

7 Senderos del Río Palancia desde Sagunto a Puerto de...

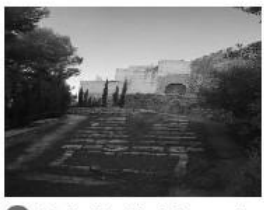

10 Geolocalización de lugares de interés en turismo cultural

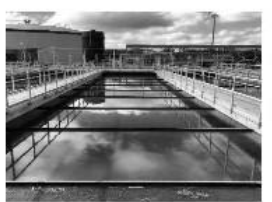

2. Buckman Waste Water

Treatment Facility....

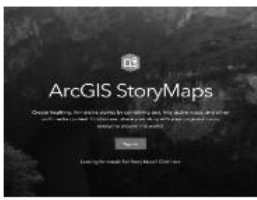

(5) How to create ArcGIS Story Maps

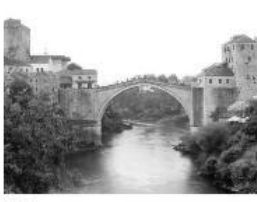

8 Mostar, One City, One River, One Bridge...Two...

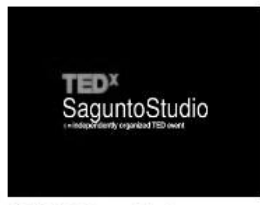

(11) TEDxSaguntoStudio

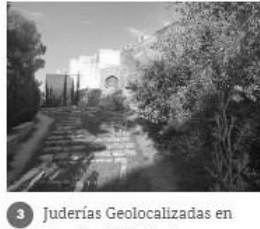
Juderías Geolocalizadas en
España $\# 100 J u d e r i a s$

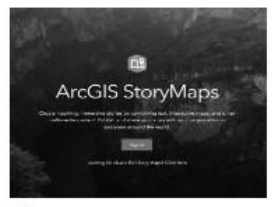

(6) Como crear ArcGIS StoryMaps

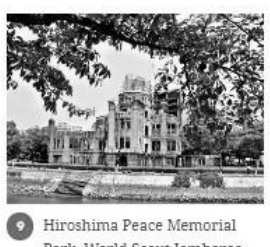

Park. World Scout Jamboree.

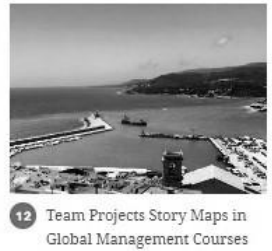

Fig. 1. Colección de Story Maps relacionados con algunos de los tópicos de los cursos.

\subsection{El Modelo de Aceptación Tecnológica TAC (Technology Acceptance Model).}

El modelo utilizado para la evaluación de ArcGIS Maps es el modelo de Aceptación Tecnológica TAC (Technology Acceptance Model) el cual sirve para determinar la aceptación en cuanto facilidad de uso y utilidad de uso de nuevas tecnologías. En los cursos de Gestión de Negocios Internacionales y Gestión Estratégica se ha utilizado este modelo para evaluar el uso de ArcGIS StoryMaps para las presentaciones de proyectos en los mencionados cursos.

El modelo de aceptación de la tecnología (Davis, 1989) ha sido uno de los modelos más influyentes de aceptación de la tecnología, con dos factores principales que influyen en la intención de un individuo de utilizar la nueva tecnología: la facilidad de uso percibida y la utilidad percibida. La utilidad percibida (PU) describe el grado en el que una persona cree que su desempeño laboral puede mejorarse mediante el uso del sistema, por ejemplo, si el sistema puede ayudarlo a completar una tarea más rápidamente. La facilidad de uso percibida (PEU) se refiere a la medida en que una persona tiene que hacer un esfuerzo mental o físico para utilizar la tecnología. 
Durante el curso piloto y al inicio del primer proyecto final en ArcGIS Story Maps, se le explicó a los estudiantes los requisitos del proyecto, la secuencia de aprendizaje y uso de esta herramienta, y el modelo para evaluar su utilidad y facilidad de uso.

\subsection{Requisitos comunes de los proyectos de los cursos de Gestión de Negocios Internacionales.}

El curso de Gestión de Negocios Internacionales tiene un proyecto con requisitos comunes a otros cursos y requisitos específicos. Los requisitos comunes son aquellos relacionados a la integración de objetivos de aprendizaje de los cursos con los objetivos de aprendizajes de los programas. Los requisitos comunes son los siguientes:

Las instrucciones de cada proyecto indican que el proyecto de equipo debe entregarse, compartirse, presentarse, y discutirse mediante una presentación elaborada en ArcGIS Story Map. No hay presentación en PowerPoint ni reporte en Word. El producto final es una presentación inmersiva e interactiva que incluye fotos, mapas, textos, gráficos y videos la cual es compartida en la plataforma de ArcGIS Story Maps. Para integrar y compartir todas las presentaciones se creó un grupo online. "Global Story Maps Campus" es un área para mostrar Story Maps creados como proyectos de equipo por los estudiantes los cuales fueron compartidos en otra colección de Story Maps (Fig. 2) (https://arcg.is/18m1nD).

Todos los miembros del equipo deben aprender a hacer un Story Map para colaborar con el proyecto del equipo. Un miembro debe crear el Story Map final para recopilar todas las fotos, enlaces, textos, etc. proporcionados por los miembros del equipo. Los miembros del equipo deben crear una cuenta pública en ArcGIS Story Maps (https://storymaps.arcgis.com). Los estudiantes pueden comenzar a practicar tan pronto como comience este curso. Se compartió un breve video de capacitación en el que muestra cómo crear un Story Map (https://youtu.be/JnhKo8ubSPM) (En las Referencias se incluye el tutorial en Español). Estos requisitos serán incorporados a los cursos una vez que ArcGIS Story Maps sea implementado en los mismos. 
para proyectos de cursos de gestión de negocios internacionales.

\section{Dr Lepervanche Global Story Maps Management Courses}

Story Maps produced by students of Supervision and Management and Business Administration programs.

Get started

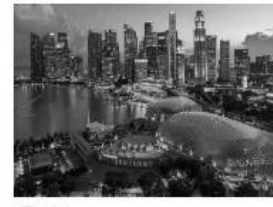

(1) Singapore

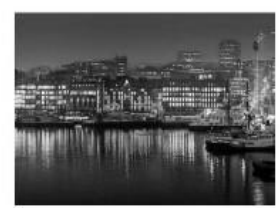

(4) Business in Norway

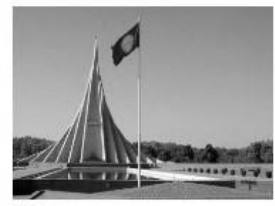

BANGLADESH

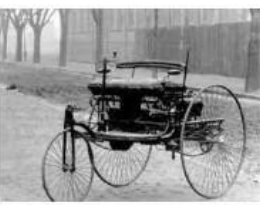

(10) Mercedes

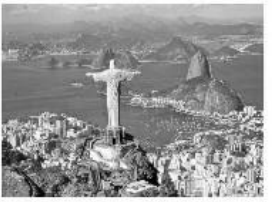

(2) Exploration of Brazil

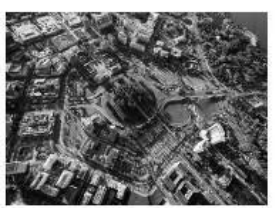

(5) Australian Consultation

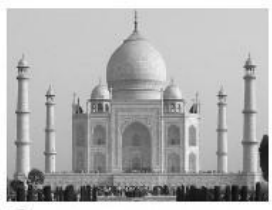

Benefits business in India

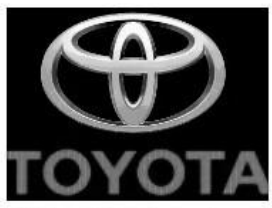

11 Toyota Motor Corporation

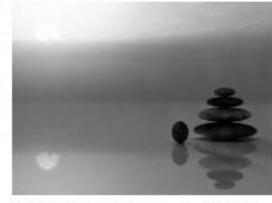

3 Business Opportunity: INDIA

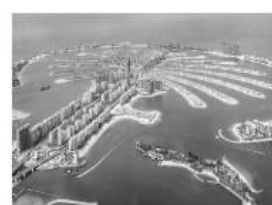

6. United Arab Emirates

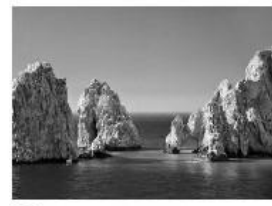

Business in Mexico

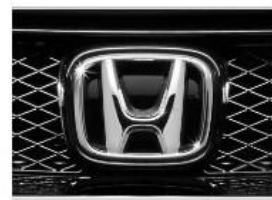

(12) HONDA

Fig. 2. Colección de Story Maps creados como proyectos de equipo por los estudiantes.

\subsection{Requisitos de los proyectos de los cursos de Gestión de Negocios Internacionales.}

El curso de Gestión de Negocios Internacionales requiere los siguientes objetivos específicos para la realización del proyecto:

Proyecto de Inteligencia de Negocios. Cada equipo tiene que explorar nuevos mercados internacionales. Deben seleccionar un país en el que crean que su cliente, una empresa estadounidense, debería abrir operaciones internacionales durante y después de la pandemia de Covid-19. Cada equipo debe producir un StoryMap de ArcGIS con texto, fotos, datos, y mapas del país que incluya:

1. Información general sobre el país seleccionado. Ubicación, capital, ciudades, bandera, recursos naturales, etc. Deben utilizar mapas y datos reales según sea necesario.

2. Análisis económico y empresarial de las condiciones actuales durante la pandemia. Cómo el país está resolviendo este problema para continuar con sus operaciones comerciales.

2. Estrategias. Desempeño actual y postura estratégica.Oportunidades y amenazas de su entorno externo y las fortalezas y debilidades de su entorno interno.

3. Culturas sociales, políticas y locales que pueden beneficiar o no a las empresas estadounidenses.

4. Otros beneficios como financieros, ambientales, alianzas, otras empresas de la misma industria, etc.

5. Evaluación de riesgos y amenazas a empresas estadounidenses. 
6. Diapositiva final, resumen sobre por qué cada equipo recomienda este país para operaciones internacionales durante y después de la pandemia de Covid-19. Qué medidas se están tomando para proteger a sus empleados y a la organización.

Este proyecto se utilizó para la evaluación inicial del uso de ArcGIS StoryMaps y se utilizó el modelo de Aceptación Tecnológica TAC (Technology Acceptance Model) para determinar la aceptación en cuanto facilidad de uso y utilidad de su uso para presentaciones de proyectos en cursos de gestión de negocios internacionales.

Los resultados, los cuales son presentados en la parte de Resultados de este trabajo, sirvieron para expandir el uso de Story Maps en otros cursos tales como los de Gestión Estratégica y sistemas de Información Global.

\section{Resultados}

Durante el curso de Gestión de Negocios Internacionales se utilizó el Modelo de Aceptación de Tecnología (TAM) el cual se utiliza para evaluar la aceptación de la tecnología por parte del usuario en términos de utilidad y facilidad de uso. El modelo TAM se ha utilizado ampliamente para predecir la aceptación del usuario. Se elaboró una encuesta la cual se centró en la aceptación por parte de los usuarios del uso de ArcGIS Story Maps para organizar y presentar el curso Proyecto de equipo para la gestión empresarial internacional. Los datos fueron recogidos mediante un cuestionario que fue rellenado durante la última semana del curso por estudiantes matriculados en los cursos en FSCJ en el semestre. La elección de esta muestra obedece a la conveniencia de su uso y a que son estudiantes activos. La última semana del curso también coincide con la semana de presentación de los proyectos a los compañeros a fin de recibir retroalimentación. Los datos se recogieron al finalizar cada curso. Los cuestionarios válidos fueron 40 .

Una de las preguntas era averiguar cómo fue el proceso de aprendizaje para utilizar ArcGIS Story Maps como herramienta para el proyecto de su equipo. El $5 \%$ de los estudiantes respondió que era muy fácil de aprender, el $40 \%$ dijo que era fácil, el $30 \%$ dijo que no era fácil ni difícil y solo el $20 \%$ dijo que era difícil de aprender (Fig. 3). 
Integración de mapas, fotos, videos e historias en ArcGIS StoryMaps

para proyectos de cursos de gestión de negocios internacionales.

The Technology Acceptance Model (TAM) is used to evaluate user

technology acceptance in terms of usefulness and ease of use. The TAM model has been widely used to predict user acceptance. This survey is about user acceptance of the use of ArcGIS Story Maps to organize a present the Team Project for International Business Management course. How was the learning process to use ArcGIS Story Maps as tool for your team project?

Answered: 40 Skipped: 0

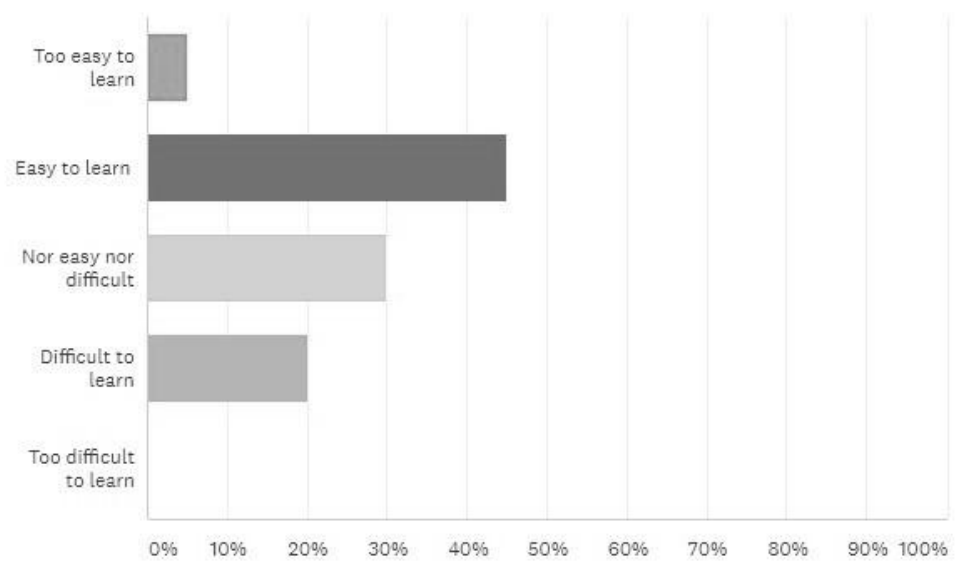

Fig. 3. Proceso de aprendizaje para utilizar ArcGIS Story Maps como herramienta para el proyecto del equipo.

La siguiente pregunta fue descubrir la facilidad después de aprender los conceptos básicos. La pregunta de qué tan fácil o no era usar ArcGIS Story Maps como herramienta para completar el proyecto de su equipo fue respondida para descubrir esta parte. El 72.5\% respondió que era muy fácil de usar, el 7.5\% que era demasiado fácil de usar, y el $12.5 \%$ que no era fácil ni difícil. El 5\% dijo que era difícil de usar y el $2.5 \%$ dijo que era muy difícil de usar. (Fig. 4). 
Once you learned the basics, how easy or not was to use ArcGIS Story Maps as tool to complete your team project?

Answered: 40 Skipped: 0

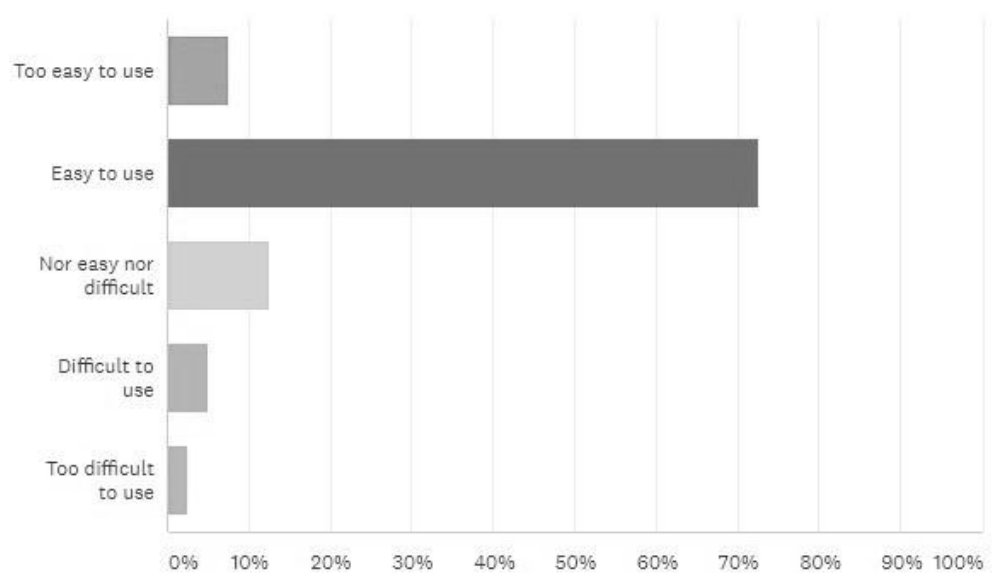

Fig. 4. Facilidad después de aprender los conceptos básicos para completar el proyecto del equipo.

Se agregó la última pregunta de la encuesta para evaluar la utilidad de la herramienta. La pregunta: una vez que completó y publicó el proyecto en ArcGIS Story Maps, ¿qué tan útil fue o no usar ArcGIS Story Maps como una herramienta para completar el proyecto de su equipo? Concluyó la encuesta. El 35\% respondió que fue muy útil, el $37.5 \%$ que fue útil y el $117.5 \%$ fue neutral. El 7.5\% dijo que fue no útil y el $2.5 \%$ que no fue muy útil (Fig. 5).

\section{Once you completed and published the project in ArcGIS Story Maps, how useful or not was to use ArcGIS Story Maps as tool to complete your team project?}

Answered: 40 Skipped: 0

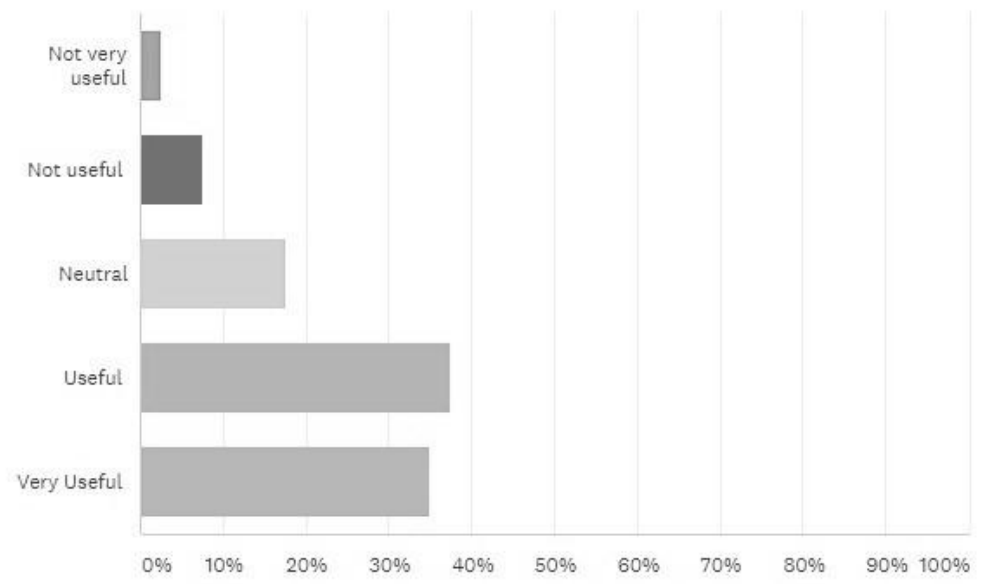

Fig. 5. Utilidad de la herramienta para completar y presentar los proyectos.

2021, Universitat Politècnica de València

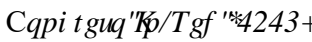


Integración de mapas, fotos, videos e historias en ArcGIS StoryMaps

para proyectos de cursos de gestión de negocios internacionales.

\section{Conclusiones}

A partir de que sólo una minoría de los estudiantes respondió que esa herramienta era difícil de aprender y que la mayoría de los estudiantes consideraba que la nueva tecnología era útil para organizar y presentar el proyecto en equipo, se tomó la decisión de ampliar el uso de esta herramienta a otro cursos con una mayor complejidad en los requisitos del proyecto de equipo. El curso seleccionado fue Gestión Estratégica. Los resultados de ambos cursos muestran la aceptación de ArcGIS Story Maps para la elaboración y presentación de proyectos en los cursos.

El curso de Gestión Estratégica comenzó con la presentación de los nuevos requisitos del proyecto para su organización y presentación en ArcGIS StoryMaps. Las interacciones iniciales presentaron las mismas dudas y preguntas que el curso anterior y la calidad de los proyectos del segundo curso superan a los del primer curso. Los proyectos de ambos cursos pueden ser visualizados en la colección de Story Maps de proyectos del semestre "Spring 2021" mostrados anteriormente en la (Fig. 2) (

La siguiente fase de este trabajo comparará y contrastará los proyectos en ambos cursos y propondrá la utilización de esta herramienta como recurso tecnológico para soportar el aprendizaje en otros cursos que requieran la integración de imágenes, videos, historias y mapas para mejorar la instrucción de estos cursos en un ambiente de aprendizaje donde el uso de mapas y visualización de datos es importante para cumplir los objetivos de aprendizaje de los respectivos programas.

\section{Referencias}

ARRIBAS, M. (2019). "Labor impagable de «geolocalizar» juderías y situarlas en internet" en ( O/ HDDQUA HO 0 HFDQWOODOPFLQR] 19 de Octubre de 2019.

Davis, F. D. 1989. 3 HFHYHG XWHXФHW SHFHYHG HDHRI XWH DQGXWHDFFHWDQHRI LQRLP DURQ UFKQRQDJ . MIS Quart. 13 319-339.

ESRI. (2012). Telling Stories with Maps A White Paper. Story Maps and ArcGIS.

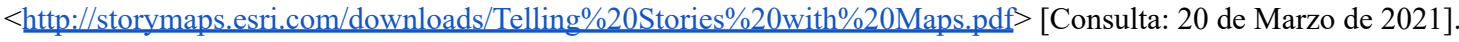

ESRI (2021). \$ LF* , 6П6 URULD DSW < https://storymaps.arcgis.com>

HWAN, R. (2017). Modern cartography and the 3D Map Revolution | Rachel Hwang | TEDxPenn. $\varangle R X 7 X E H$

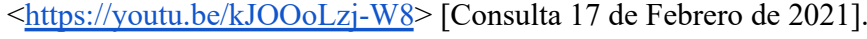

LEPERVANCHE, J. (2018). "Integration of TED-Ed Lessons and TED and TEDx Talks to Enhance College Classroom Instruction" en Fourth International Conference on Higher Education Advances HEAd'18. $<$ https://www.researchgate.net/publication/326501714 Integration of TED-Ed Lessons and TED and TEDx Talks to Enhance College Classroom_Instruction> [Consulta: 19 de marzo de 2021].

LEPERVANCHE, J. (2021). Como Crear ArcGIS StoryMaps Tutorial / Dr. Jose G. Lepervanche Valencia. $\varangle R X 7 X E H$

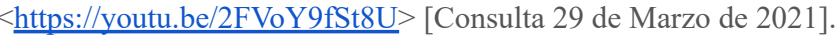

LEPERVANCHE, J. (2021). Dr. Lepervanche Global Learning Maps Collection $<\underline{\mathrm{https}}: / / \operatorname{arcg}$.is $/ 15 \mathrm{aeSu} 0>$ [Consulta 29 de Marzo de 2021].

LEPERVANCHE, J. (2021). Dr Lepervanche Global Story Maps Campus in ArcGIS. <https://arcg.is/Le15b> [Consulta 29 de Marzo de 2021].

LEPERVANCHE, J. (2021). How to create ArcGIS StoryMaps tutorial / Dr. Jose G. Lepervanche Valencia. $\varangle R X 7 X E H$

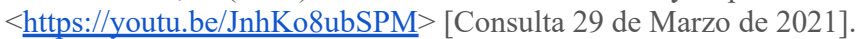

McDERMOTT, T. (2017). For Civic Good | Twyla McDermott | TEDxHickory. $<R X 7 X E H$ $<$ https://youtu.be/GOPURFcKU1Q>. 